\title{
Práctica de valores apoyada en las tecnologías de la información y la comunicación como estrategia para la sana convivencia ${ }^{1}$ \\ Values practice supported by information and communication technologies as a strategy for healthy coexistence
}

DOI: http://dx.doi.org/10.17981/cultedusoc.9.3.2018.73

Artículo de investigación. Fecha de recepción: 15/06/2018. Fecha de aceptación: 27/11/2018

\author{
Angela Campo Parodis; Anibal Sierra Ospino; Bleydis Barrios Caceres; \\ Carmen Caballero Contreras; Carmen Bermudez Tapias; Catia Orozco Orozco; \\ Diana Barrios De Aguas; Dilia Contreras Aragon; Edelfin Aragon Arrieta; \\ Exel Orozco Barrios; Fabiola Contrera Delgado; Fidelina Medina Lizcano; \\ Gladis Perea Fontalvo; Guillermo Acuña Anaya; Hector Ochoa Agamez; \\ Hugo Diaz Caballero; Jaimar Aleman Barrera; Jose Carvajal Mercado; \\ Jhovannys Barrios Peña; Julia Delgado Padilla; Katia Delgado Pacheco; \\ Lidis Garcia Barreto; Manuel Silva Andrade; Maria Rambal De La Cruz; \\ Rosmiry Orozco Meriño; Rubys Lidueña Escobar; \\ Seneth Montero Herrera y Urith Anaya Orozco ${ }^{2}$ \\ Institución Educativa Departamental Santa Rosa de Lima. Magdalena (Colombia)
}

Para citar este artículo:

Campo, A., Sierra, A., Barrios, B., Caballero, C., Bermudez, C., Orozco, C., Barrios, D., Contreras, D., Aragon, E., Orozco, E., Contrera, F., Medina, F., Perea, G., Acuña, G., Ochoa, H., Diaz, H., Aleman, J., Carvajal, J., Barrios, J., Delgado, J., Delgado, K., Garcia, L., Silva, M., Rambal, M., Orozco, R., Lidueña, R., Montero, S. y Anaya, U. (2018). Práctica de valores apoyada en las tecnologías de la información y la comunicación como estrategia para la sana convivencia. Cultura. Educación y Sociedad 9(3), 619-626. DOI: http://dx.doi.org/10.17981/ cultedusoc.9.3.2018.73

\section{Resumen}

El presente artículo muestra los resultados de una experiencia investigativa realizada tomando en cuenta las problemáticas existentes en la Institución Educativa Departamental (IED) Santa Rosa de Lima. Surge la idea de este proyecto de investigación apoyado en los aportes de las tecnologías de la información y la comunicación (TIC), y pretende fomentar la práctica de valores, apoyados en las tecnologías de la información y la comunicación como estrategia para la sana convivencia. Se trabajó bajo la metodología cualitativa con modelo de investigación acción participativa. La población estuvo conformada por estudiantes de primaria de la Institución Educativa Departamental (IED) Santa Rosa de Lima. Para recoger los datos se utilizaron, encuestas y observación directa. Se evidenció que aspectos como la percepción del entorno y conductas inapropiadas realizadas por los estudiantes, conllevan a la permanencia de la mala convivencia en la institución.

Palabras clave: Tecnologías de la Información y la Comunicación (TIC), estrategias pedagógicas, formación en valores, convivencia

\section{Abstract}

This article shows the results of a research experience carried out taking into account the existing problems in the Departmental Educational Institution (IED) Santa Rosa de Lima. The idea of this research project is based on the contributions of information and communication technologies (ICT), and aims to promote the practice of values, supported by information and communication technologies as a strategy for healthy coexistence. We worked under the qualitative methodology with participatory action research model. The population was conformed by students of primary of the Educational Institution Departmental (IED) Santa Rosa de Lima. To collect the data were used, surveys and direct observation. It was evidenced that aspects such as the perception of the environment and inappropriate behaviors carried out by the students, lead to the permanence of the bad coexistence in the institution.

Keywords: Information and Communication Technologies (ICT), pedagogical strategies, training in values, coexistence

1 Este artículo ha sido derivado del Programa de Fortalecimiento de la Cultura Ciudadana y Democrática CT+I a través de la IEP apoyada en TIC en el Departamento de Magdalena: CICLON.

22 Docentes miembros del grupo de investigación "Vadisco" de la institución Educativa Departamental Santa Rosa de Lima-Magdalena.

- The author; licensee Universidad de la Costa - CUC.

Cultura, Educación y Sociedad vol. 9 no. 3, pp. 619-626. Diciembre, 2018

Barranquilla. ISSN 2389-7724 Online 


\section{Introducción}

La convivencia es una temática de suma importancia para la tranquilidad de la sociedad en general (Bolívar y Balaguer, 2007). En las instituciones educativas se hace imperante mantener una sana convivencia, basada en la vivencia de los valores y normas que la fomenten. En la Institución Educativa Departamental (IED), Santa Rosa de Lima, Magdalena, se ha manifestado un incremento en el comportamiento inadecuado por parte de los estudiantes, lo cual ha generado dificultad en la convivencia de la comunidad estudiantil. Esta problemática ha sido evidenciada a través de la observación directa y por manifestación de los padres de familia quienes indican que este comportamiento es similar en sus hogares.

Así mismo, se evidencia falta de interés y compromiso por el estudio por parte de los estudiantes; ausentismo escolar, conflictos entre compañeros, violencia verbal y física, poca higiene personal y falta de sentido de pertenencia con la institución (Aron y Milic, 1999; Chaux, 2012).

Si bien se han acudido a estrategias como firma de actas de compromiso de estudiantes y padres de familia, esto no ha sido lo suficiente para disminuir las conductas de indisciplina de los estudiantes, lo cual sugiere que se utilicen otros métodos que contribuyan a mejorar la convivencia escolar para que todos aprendan y se sientan bien tratados (Mena, 2007; Mockus, 2002). Por todo lo expuesto, se hace necesario y pertinente realizar este proyecto de investigación, con el fin mejorar estos aspectos que están ocasionando dificultades en distintos ámbitos de los estudiantes, apoyando lo anterior en las familias y la comunidad.
Los docentes de la IED Santa Rosa de Lima, conforman un grupo de investigación con el objetivo de disminuir esta problemática utilizando herramientas que le fuesen útiles y acertadas para trabajar con los estudiantes. Las tecnologías de la información y la comunicación (TIC), son una herramienta valiosa que permite un acercamiento importante con los estudiantes ya que por medio de estas interactúan y perciben las temáticas de una manera más didáctica. Al hablar de la incorporación de las TIC mencionamos necesariamente cómo ha cambiado el acceso a la información, la diversidad de escenarios que diversifican las modalidades de información y satisfacen la diversidad de estilos de aprendizaje e inquietudes que se le presentan a los actores del proceso educativo. Todo esto proporciona alternativas al docente para facilitar el diseño de experiencias que debe contextualizar a las demandas y expectativas del alumno, para hacerlas más motivantes y significativas, no sólo en el caso de las instituciones educativas, sino en wl campo comunitario, político, religioso, económico, cultural, étnico y comunicacional en general (Herrera, 2016; Cabero, 2012). Para Cacurri (2013), cada día aumentan la cantidad de docentes que utilizan herramientas informáticas, y no solo en los procesos de enseñanza aprendizaje de saberes académicos, sino también en el diseño de experiencias para formarse en valores y el mejoramiento de la calidad de vida actual y futura.

\section{Metodología}

El artículo que se presenta es un producto derivado del estudio práctica de valores apoyado en las tecnologías de la información y la comunicación como estrategia para la sana convivencia. Se 
desarrolló en la Institución Educativa Departamental (IED) Santa Rosa de Lima; su objetivo principal ha sido afianzar los valores en los estudiantes como estrategia para mejorar la convivencia en la institución.

El estudio contempla una metodología el orden secuencial siguiente: En el diseño se trabajó con la metodología cualitativa, la cual estudia la realidad en su contexto natural, inviviéndose en los hechos tal y como suceden, intentando extraer el sentido de lo vivido e interpretar los fenómenos de acuerdo con los significados que tienen para las personas implicadas (Ruiz, 2012; Pérez, 2002; Hernández 2014). Se utilizó el modelo de investigación acción participativa, porque es una propuesta metodológica que involucra a la comunidad en el conocimiento, intervención y solución de sus problemas. En este sentido, se desarrollan acciones que no sólo busca solamente describir los problemas sino, generar conjuntamente con la comunidad los conocimientos y las alternativas necesarias para definir las acciones adecuadas que estén en la línea la transformación necesaria y pertinente (Schutter,1987; Báez, 2009; Martínez, 2004).

La unidad de análisis estuvo conformada por estudiantes de primaria de la Institución Educativa Departamental (IED) Santa Rosa de Lima. Para recoger los datos se utilizaron, encuestas y observación directa. Así mismo, se utilizó una adaptación del cuestionario de convivencia de la I.ES Miguel Catalán diseñado por Miguel Vaquero Herera en España.

Entre los instrumentos, se trabajó con la observación directa, porque "Observar supone una conducta deliberada del observador, cuyos objetivos van en la línea de recoger datos en base a los cuales poder formular o verificar hipótesis"
(Fernández- Ballesteros, 1980, p. 135). En este estudio, se trabajó la observación para recoger la información detallada de la situación que presenta el objeto de estudio, permitió el registro riguroso y la descripción de las situaciones que procesamos, de acuerdo a las intenciones de búsqueda que nos planteamos.

También utilizamos como instrumento la encuesta, porque permite indagar las opiniones, posturas, actitudes y comportamientos de las personas que seleccionamos como muestra representativa para conocer a profundidad sobre el tema de estudio (Eyssautier, 2006).

\section{Resultados}

Se evidenció que aspectos como la percepción del entorno y conductas inapropiadas realizadas por los estudiantes, conllevan a la permanencia de la mala convivencia en la institución.

Sobre la percepción del entorno

1. El $2 \%$ se ha sentido solo en la institución. El 60 \% de los estudiantes se lleva bien con sus compañeros de clase.

2. El $70 \%$ se lleva bien con los docentes.

3. El $70 \%$ de los estudiantes creen que sus compañeros tienen una buena impresión de ellos.

4. El $70 \%$ creen que los docentes tienen una percepción "Normal" de ellos.

5. El $50 \%$ de los compañeros ha insultado a sus compañeros alguna vez.

Conductas inapropiadas que el estudiante realiza.

En cuanto a las pregunta: ¿Has insultado a algún compañero/a?: ¿Has insultado a algún compañero/a? Las respuestas giraron en torno a "Siempre" y "A menudo": el 60 \%.

Sobre la pregunta: ¿Te han insultado los compañeros/as?, las respuestas estuvieron entre "Siempre" y "A menudo" en un $40 \%$. 
En general, el alumnado tiene mayor tendencia a insultar que a ser insultado.

En cuanto a la pregunta: ¿Has pegado, empujado a un compañero/a? a un compañero/a? Las respuestas estuvieron entre"Siempre" y "A menudo", en un $61 \%$.

A la pregunta: ¿Te han pegado o empujado? Las respuestas señalaron entre "Siempre" y "A menudo", en un $35 \%$.

En general el alumnado tiene mayor percepción de pegar o ser empujado a recibirlo.

Ante la pregunta: ¿Has insultado a algún compañero/a? . Las respuestas fueron: "Siempre" y "A menudo", en un $73 \%$.

En cuanto a la pregunta: ¿Te han insultado los compañeros/as? . Las respuestas se dieron entre "Siempre" y "A menudo", en un 55\%. La frecuencia de insultos entre alumnos/as está entre “ Bastante" y "Mucho", en un 56,2 \%.

Sobre la frecuencia de acciones inadecuadas, las cifras s expresaron como sigue:

- La frecuencia de peleas entre estudiantes es de $40 \%$.

- La frecuencia de los grupos que no se llevan bien dentro de la clase es de $30 \%$.

- La frecuencia de hostigamiento e intimidación entre estudiantes es de $40 \%$

- La frecuencia de acoso sexual en los estudiantes es de $6 \%$.

- La frecuencia de miedo de estudiantes a otros es del $20 \%$.

\section{Conclusiones}

De acuerdo a los resultados obtenidos en el presente proyecto se evidencia la necesidad de continuar trabajando en el fomento de los valores como estrategia para mejorar la convivencia estudiantil y, por ende, en la convivencia de la sociedad en general. Si bien se identificaron las causas de la problemática y se implementaron nuevas estrategias pedagógicas apoyadas en TIC, para fortalecer las debilidades encontradas, se evidencia la necesidad de crear programas enlazados o encardinados a lo largo del currículo escolar, que permitan de manera transversal fortalecer los valores como pilar fundamental en el comportamiento de los estudiantes. De igual forma los padres o cuidadores son una parte fundamental para la consecución del objetivo principal que es mejorar los comportamientos desadaptativos que irrumpen en la dinámica escolar, familiar y social por parte de los estudiantes (De la Concepción, 2015).

Es evidente entonces que lo que se busca es enfatizar en la relevancia que tienen las estrategias pedagógicas para el mejoramiento del proceso de enseñanza de la sana convivencia a través de las TICS como parte de su formación, donde el aprendizaje sea autónomo, critico, constructivo y más atractivo para los estudiantes si lo hacen a través de este tipo de herramientas. Con referencia a lo anterior, en el desarrollo de experiencias con las TICS, se logra determinar que efectivamente a los estudiantes cuando se les suministra una educación con herramientas tecnológicas, evidencian un notable interés por este tipo de prácticas pedagógicas, por lo cual, no se trata de continuar con la educación tradicional si no de enriquecer la práctica pedagógica con una visión más actualizada de la enseñanza, para atraer la atención y el interés de los niños y jóvenes y así mejorar la calidad educativa.

En este sentido, se hace necesario atender lo planteado por Ausubel (2006), abanderado de la teoría del aprendizaje significativo, quien presenta orientacio- 
nes para hacer más significativo el aprendizaje para el alumno, que es realmente la intención última de la tarea educativa, lograr que ocurra el aprendizaje. Para lograr el desarrollo integral del alumno, la experiencia que se promueva debe asociarse a situaciones significativas para el alumno, la incorporación de los nuevos conocimientos y nuevas aplicaciones que desarrollen sus habilidades y competencias comunicacionales, deben contextualizarse en los acontecimientos que le sean significativos, para que tengan experiencias con sentido, conscientes de los logros alcanzados y con compromiso por seguir aprendiendo e interviniendo en esa realidad. Cuando se enseñan contenidos, como es el caso de las ciencias naturales, u otras disciplinas científicas, los estudiantes deben vivir la experiencia de manera consciente, e ir alcanzando habilidades y competencias que reflejan su evolución y su inserción en el contexto en el cual cursa sus estudios, de manera pertinente a su desarrollo, a sus intereses y a la realidad; esto hará que se desarrolle el deseo de seguir aprendiendo (Ochoa, 2011).

Dadas las orientaciones que anteceden, resulta indispensable que la tarea educativa se contextualice en los escenarios actuales y que anticipe las condiciones futuras, es necesaria una mirada a la prospectiva de la evolución y los criterios de calidad en el cual tendrán que actuar los estudiantes. Es importante que se satisfagan las necesidades de las generaciones futuras, por estas razones es necesario el uso de estrategias didácticas aprovechando los beneficios de las TIC, que se promuevan experiencias que permitan la creación de una sana convivencia entre los estudiantes de la comunidad educativa, debido a que se le permite así al estudiante no solo la exploración de posibles soluciones, la mode- lación de la realidad, sino el desarrollo de estrategias y la aplicación de técnicas que le permitan desarrollarse en su propia cotidianidad académica (Ochoa, 2011).

Según lo expuesto por el MEN en el 2004, planteado en la guía $\mathrm{n}^{0}$ 7: Se ratifica la necesidad de una formación dirigida a que los estudiantes desarrollen una cultura científica y tecnológica, que les permita aproximarse, comprender e intervenir la realidad y sea capaz de avizorar sus demandas a futuro. Este es un lineamiento que guía las instituciones educativas del país.

En ese orden de ideas, se es pertinente resaltar que la vida de hoy de los estudiantes se lleva a cabo en diversos contextos partiendo de lo multicultural e interconectado de la sociedad. Al respecto, Demarchi, Aguirre, Yela y Viveros (2015), puntualizan que la dinámica familiar resulta clave, para comprender las interacciones que se dan entre los diferentes miembros de forma tal que se fortalezca su plano personal y se incida en el desarrollo de relaciones afectivas $\mathrm{y}$ habilidades para vivir en sociedad. Este hecho es lo que logra exigirles a los sistemas educativos el poder orientar la educación para el desarrollo de habilidades, capacidades cognitivas, competencias, aptitudes, actitudes y valores que posibiliten a los estudiantes el poder de actuar en ambientes abiertos que exigen la utilización y la adquisición de las grandes mejoras de las Tecnologías de la Comunicación y de la Información. Hechas las consideraciones anteriores dentro de este contexto, es verídico que las tecnologías de la información, están siempre al servicio de la educación puesto que a la comunidad educativa le ofrece dinamismo, interactividad, almacenamiento y procesamiento de la información multidisciplinarmente. 
Se hace necesario intervenir y cambiar la práctica de la enseñanza, formarse en el manejo de herramientas TIC y aprovechar las habilidades de los estudiantes; a la vez promover experiencias de aprendizaje significativo en las diversas áreas del conocimiento. La incorporación de las TIC en el fortalecimiento de la motivación por aprender, tiene ventajas, lo más importante es que esas ventajas sean aprovechadas para formar vínculos, en indagar y vivir el desarrollo de tareas colaborativas, generar co-responsabilidad y comprensión de la diversidad en la participación activa, desarrollar la capacidad de conocer nuevos y variados escenarios, establecer relaciones, interpretar pensamientos, culturas y posturas diferentes. Este es otro lineamiento curricular del MEN en Colombia (2004).

En este sentido, es pertinente que en cada uno de los participantes sientan la motivación, se disponga a la integración con la comunidad y al desarrollo de ciertas habilidades intelectuales y de convivencia; tales como el razonamiento, la resolución de problemas, la creatividad y la capacidad de aprender a aprender y convivir en cualquier contexto social. Es necesario que con las TIC los estudiantes puedan aprovechar el tiempo libre dentro y fuera de las aulas; permitiendo el acercamiento a la praxis de valores sociales, convivenciales, culturales, deportivos y ciudadanos, donde el estudiantado aprenderá saberes y rescatará valores desde la acción de su entorno escolar.

En el tema de la creatividad entendida desde el uso de las TIC como medios de comunicación, según Nuñez y Ravina (2017), la misma se fundamenta en la originalidad, la capacidad de adaptación a la realidad y el grado de elaboración. Con el desarrollo de las TIC que ha penetrado casi todas las actividades y ámbitos, tanto públicos como privados, se han desafiado las prácticas pedagógicas; lo importante es no considerar como enemigos los estímulos de la televisión $\mathrm{y}$ el internet, tan frecuentes en la cotidianidad de los estudiantes, sino como unos aliados para motivar y proponer experiencias innovadoras en la formación de niños y jóvenes. Así mismo sucede con la incorporación de los servicios que ofrece el teléfono celular, el internet y otras manifestaciones actuales como la computadora, los videojuegos, los reproductores, etc. (Ochoa, 2011).

En el mundo de hoy, con el auge de TIC, las instituciones educativas y las organizaciones sociales deben desarrollar vínculos importantes en proyectos de aprovechamiento de la sociedad del conocimiento, para el desarrollo pertinente de las personas y la sociedad en general. Se requiere comprender dos cosas: Una es que las TIC son relevantes en la medida que logren fomentar maneras de conocer, reutilizar, pensar y actuar. Segundo, es necesario en la actualidad, aprovechar la oportunidad de vincular los escenarios, sus culturas y las realidades, la forma cómo resuelven sus diversas problemáticas, las fortalezas propias delos contexto, las oportunidades y las amenazas globales que se presenten; al tiempo que es importante enseñar a los estudiantes a descifrar los contenidos expuestos en los medios y siempre valorar la calidad en función de su desarrollo humano y social sostenible.

\section{Referencias}

Arón, A. y Milic, N. (1999). Clima social escolar y desarrollo personal. Un programa de mejoramiento. Santiago de Chile: Editorial Andrés Bello. 
Báez, J. (2009). Investigación cualitativa. Madrid: ESIC Editorial.

Bolívar, A. y Balaguer, F. (2007). La Educación para la Ciudadanía: marco pedagógico y normativo. Recuperado de http://www. educacionenvalores.org/IMG/pdf/ bolivar_y_balaguer.pdf

Cabero, J. (2012). El rol del profesor ante las nuevas tecnologías de la información y la comunicación. Agenda académica, 7(1), 41-57.

Cacurri, V. (2013). Revista Educación con TIC, nuevas formas de enseñar en la era digital. Buenos Aires.

Chaux, E. (2012). Educación, convivencia y agresión escolar. Bogotá, D.C.: Taurus.

De la Concepción, A. (2015). Concepciones sobre participación de niñas, niños y adolescentes: Su importancia en la construcción de la convivencia escolar. Cultura Educación y Sociedad, 6(2), 9-28. Recuperado de https:// revistascientificas.cuc.edu.co/culturaeducacionysociedad/article/download/840/pdf_105/

Demarchi, G., Aguirre, M., Yela, N. y Viveros, E. (2015). Sobre la dinámica familiar. Revisión documental. Cultura Educación y Sociedad, 6(2). 117138. Recuperado de https:// revistascientificas.cuc.edu.co/culturaeducacionysociedad/article/ view/1049/pdf_223

Eyssautier, M. (2006). Metodología de la investigación. México, D.F.: Cengage Learning.

Fernández-Ballesteros, R. (2005). Introducción a la evaluación psicológica. Madrid: Pirámide.

Hernández, R., Fernández, C. y Baptista, P. (2014). Metodología de la investigación. Mexico, D.F.: McGraw-Hill Education.
Herrera, A. y Herrera, A. (2016). Las TIC como herramientas pedagógicas. Aracelisherrera10.blogspot.com.co. Retrieved 9 March 2018, from http:// aracelisherrera10.blogspot.com.co/

Mena, I. (2007). Acuerdos de convivencia escolar para que todos aprendan y se sientan bien tratados. Recuperado de http://valoras.uc.cl/images/centro-recursos/equipo/FormacionDeComuni-

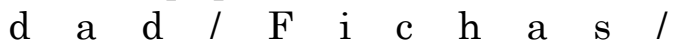
Acuerdos-de-convivencia-escolar-para-que-todos-aprendan-bien-y-sesientan-bien-tratados-2018.pdf

Martínez, M. (2004). Ciencia y arte en la metodología cualitativa. México D.F: Editorial Trillas.

Mockus, A. (2002). La educación para aprender a vivir juntos. Convivencia como armonización de ley, moral y cultura. Perspectivas, XXXII, (1). 19-37. Recuperado de http://www. ibe.unesco.org/fileadmin/user_ upload/archive/Publications/Prospects/ProspectsPdf/121s/121smock. pdf

Núñez, E. y Ravina, R. (2017). Análisis del nivel de competitividad empresarial en el panorama publicitario colombiano fundamentada en el éxito creativo y la responsabilidad ética y jurídica. JURÍDICAS CUC, 13(1). 9-28. http://dx.doi.org/10.17981/juridcuc.13.1.2017.1

Ochoa, M. (2011). Implementación de las TIC como estrategia didáctica para generar un aprendizaje significativo de los procesos celulares, en los estudiantes de Grado Sexto de la Institución Educativa San Andrés, del Municipio de Girardota. [Tesis]. Universidad Nacional de Colombia, Medellín. Recuperado de http://www. b d i g i t a l. u n a l. e d u . co/5936/1/43666105.2012.pdf 
Pérez, G. (2002). Investigación cualitativa. Retos e interrogantes. II técnicas y análisis de datos. Madrid: La Muralla.

Ruiz, J. (2012). Metodología de la investigación cualitativa. Bilbao: Universidad de Deusto.
Schutter, A. (1987). Metodo y proceso de la investigación participativa en la capacitación rural. Patzcuaro, Michoacan: CREFAL. 